\title{
Convenient near-field optical measurement and analysis of polystyrene spheres
}

\author{
Sy-Hann Chen ${ }^{\mathrm{a}, *}$, Hsu Chou Hsiung ${ }^{\mathrm{b}}$, Wei-Bin Suc ${ }^{\mathrm{c}}$, Din Ping Tsai ${ }^{\mathrm{d}}$ \\ a Department of Applied Physics, National Chiayi University, Chiayi 600, Taiwan, ROC \\ ${ }^{\mathrm{b}}$ Taiwan Branch, FEI Company, HsinChu 302, Taiwan, ROC \\ ${ }^{\mathrm{c}}$ Institute of Physics, Academia Sinica, Taipei 115, Taiwan, ROC \\ ${ }^{\mathrm{d}}$ Department of Physics, National Taiwan University, Taipei 106, Taiwan, ROC
}

Received 5 December 2005; accepted 10 January 2006

\begin{abstract}
The modification of a commercial tapping mode atomic force microscope (AFM) into a transmission mode near-field scanning optical microscope (NSOM) is presented and polystyrene spheres in the diameter of $100 \mathrm{~nm}$ are used in this experiment. The detection of nearfield signals is based on photodiodes with lock-in technique, and resolutions of topography and near-field signals obtained are about 10 and $20 \mathrm{~nm}$, respectively. Furthermore, it is discovered that the computer-simulated near-field energy distribution profile, obtained by scanning over polystyrene spheres under illumination mode, falls within 5\% range as compared with experimental values. The near-field absorption coefficient can be determined by this way. This will be a useful theoretical model to analyze the near-field transmission effect from others.
\end{abstract}

(C) 2006 Elsevier Ltd. All rights reserved.

Keywords: Atomic force microscope (AFM); Near-field scanning optical microscope (NSOM); Polystyrene spheres; Near-field absorption coefficient

\section{Introduction}

As technology advances, producing lighter, thinner and smaller electro-optic components has become a vital requirement in manufacturing processes. Similarly, the techniques of analyzing and testing components have to be upgraded to meet such requirements. Among them, the scanning probe microscopy (SPM) [1], one of the welldeveloped methods, is applied extensively to analyze the surface structure of numerous types of samples, and the working principle of which relies on a micro-fabricated tip suspended above a sample at a distance ranging from 1 to $20 \mathrm{~nm}$. When scanning over the sample, the tip-sample distance is regulated by means of shear force or normal force. A computer connected through electronics feedback system records changes in the interaction force. Based on collected data, surface topography of the sample is then built up.

\footnotetext{
${ }^{*}$ Corresponding author.

E-mail address: shchen@mail.ncyu.edu.tw (S.-H. Chen).
}

Fiber optics that allow direct transmission of light are widely used in optical communication. If replacing the above-mentioned sharp tip with optical fiber probe, acquisition of surface structure profile and near field optical signal can be carried out simultaneously. This kind of imaging method is known as near-field scanning optical microscopy (NSOM) [2-6]. Unlike the conventional optical microscopy, NSOM is capable of detecting microstructure beyond the diffraction limit of light. Moreover, it also has the advantage of being virtually nondestructive to the surface of samples. In this article, we also present a theoretical model to determine absorption coefficient of materials in microscopic condition, which greatly extends applications of nano-materials.

\section{Experimental}

In this paper, the construction of a transmission mode NSOM based on a tapping mode AFM is presented. The normal force detection head is replaced by a shear force detection [7-9] module utilizing a straight fiber probe. An 
additional circuit provides control electronics with amplification and proper interface. The configuration is transparent to the commercial AFM system and the operation is identical to the tapping mode except for the probe alignment. The detection of near-field signals is based on photodiodes and lock-in technique.

A $125 \mu \mathrm{m}$ telecommunication single mode fiber was used in this study. The straight fiber probe could be easily fabricated by a Sutter Instrument P2000. Before using in NSOM system, the fiber probe needs to be coated with a layer of Al film around the tip with transmission efficiency measured at about $10^{-3}$. For detection of the shear force, the fiber probe is first dithered at its resonance frequency and then brought to the sample. The amplitude, detected by the optical beam diffraction method [10], decreases when the distance is less than about $20 \mathrm{~nm}$, and can be used as a feedback for distance regulation.

A schematic diagram of the experimental setup is shown in Fig. 1. The AFM system used for modification is a Multimode Nanoscope II manufactured by Digital Instruments. The input diode laser light is modulated at $81.43 \mathrm{kHz}$, and then focused into the end of the fiber probe cleaved by a cleaver (FK11, Newport). To detect the near-field signal, the transmitted light is measured by a $1 \times 1 \mathrm{~cm}^{2} \mathrm{Si}$ photodiode (S100 V, UDT Sensors) underneath the sample followed by a current amplifier (SR570, Stanford Research Systems). Signal is sent to lock-in amplifiers (SR830, Stanford Research Systems) and then recorded by the AFM control unit. In Fig. 2, simultaneously acquired $1.05 \times 1.05 \mu^{2}$ images of the AFM and NSOM of $100 \mathrm{~nm}$ polystyrene spheres (Duke Scientific Corporation) on glass are presented. The resolution is about $10 \mathrm{~nm}$ for the topography, while an almost doubled value of $20 \mathrm{~nm}$ as determined from the full width at half maximum of the intensity is achieved for the near-filed signal.

\section{Theoretical analysis}

Although the near-field optical signal with high resolution can be easily obtained by the photodiode underneath the sample, there is not an ecumenical demonstration on theoretical grounds $[11,12]$. Therefore, a novel theoretical model is also submitted to analyze the near-field energy collected by a photodiode. The profile of field energy intensity distribution at the end-panel of an optical fiber can be expressed by the Fresnel approximation $[13,14]$ shown below

$I(z, r)=I_{0} \frac{\exp \left(-2 r^{2} / \omega_{0}^{2} \alpha\right)}{\alpha}$,

where $\alpha \equiv 1+\left(\lambda z / \pi \omega_{0}^{2}\right)^{2}, \lambda$ is the wavelength of incident light, $z$ is the distance when light ray traveling away from the end-panel of the optical fiber to a point along the optical axis, $r$ is the lateral displacement perpendicular to the optical axis and the spot size, $\omega_{0}$, is the radius of the
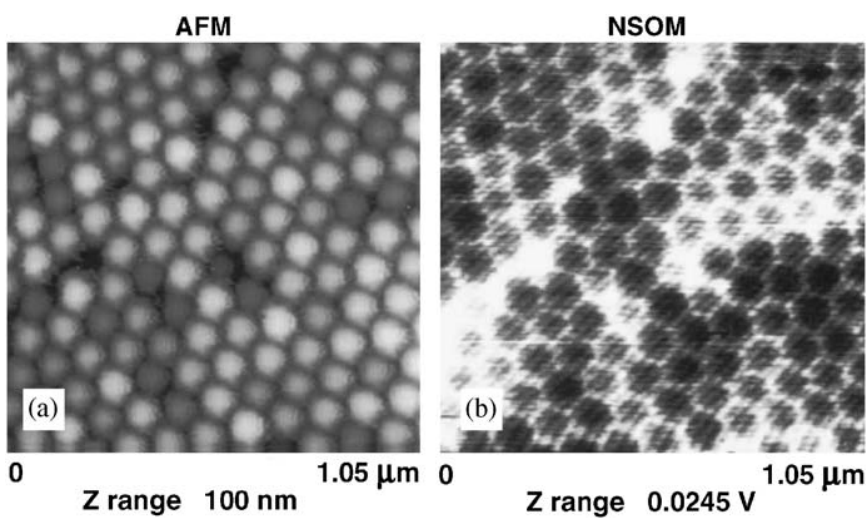

Fig. 2. Simultaneously acquired $1.05 \times 1.05 \mu \mathrm{m}^{2}$ images of (a) topography and (b) reflected light on a $100 \mathrm{~nm}$ polystyrene spheres test sample.

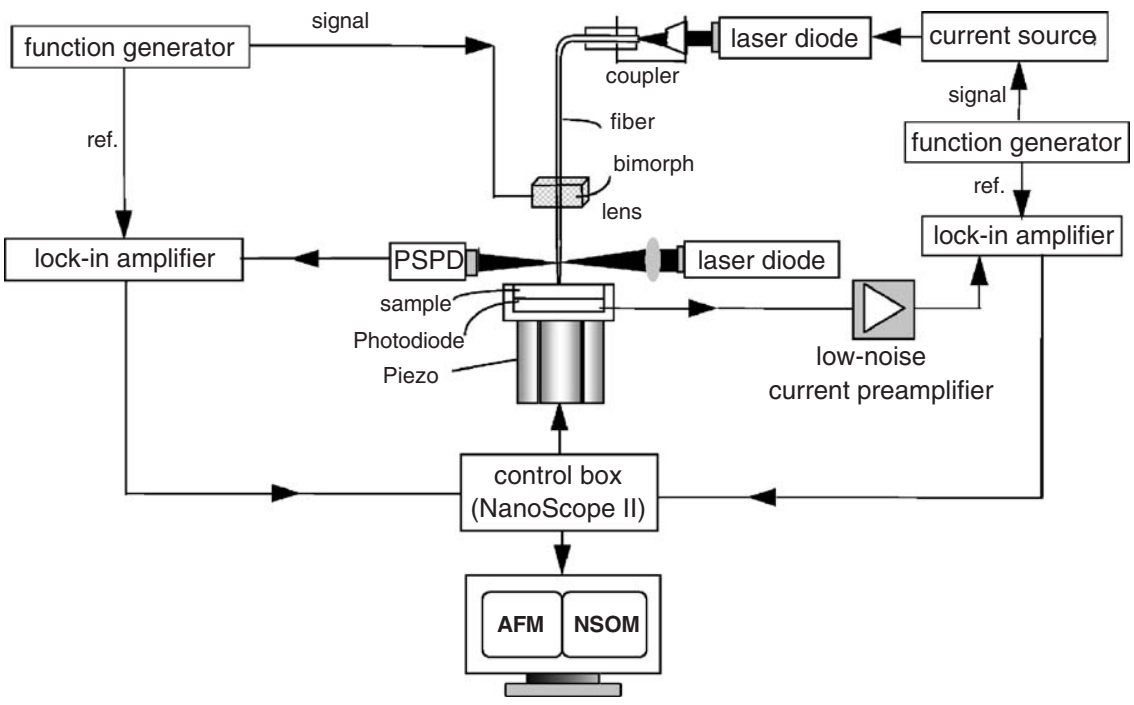

Fig. 1. Schematic diagram of the transmission mode NSOM. 
$1 / e^{2}$ irradiance contour of the mode in the fiber and at the exit face. From Butler et al. [15], it is known as 0.61 times of the aperture radius at the end-panel of optical fiber. To calculate the near-field signal intensity, the optical fiber needs to be modeled with its end formed into an apertureNSOM probe tip. According to Transmission Line Theory, as the aperture diameter of interest is much smaller than the wavelength of the incident light, all waveguide modes run into cutoff before reaching the apex. That is, wavelength of the near-filed light is equal to the diameter of this aperture hole. After replacing $\lambda$ with the aperture diameter of the fiber probe, the Eq. (1) can be rewritten as

$$
\begin{aligned}
I(z, r) & =I_{0} \frac{\exp \left[-2 r^{2} /(0.61 a)^{2} \alpha\right]}{\alpha} \text { and } \\
\alpha & \equiv 1+\left[2 a z / \pi(0.61 a)^{2}\right]^{2} .
\end{aligned}
$$

Here $a$ is the aperture radius of the fiber tip while other terms are the same as above. The tip diameter is measured to be about $20 \mathrm{~nm}$ by a scanning electron microscope. Fig. 3 indicates the simulated light intensity distribution profiles as derived from Eq. (2) for the six given positions $z / a=0.01,0.2,0.4,1.0,1.5,2.0$. Curve 1 in Fig. 3 demonstrates the highest near-filed light intensity. It occurs at close proximity of nano-aperture plane $(z \cong 0)$. As $z$ increases, not only the corresponding intensity falls considerably but also the mode-field diameter (MFD) raises substantially. It goes without saying that the nonpropagating and evanescent field [16] exists in front of the nano-aperture plane of the fiber probe.

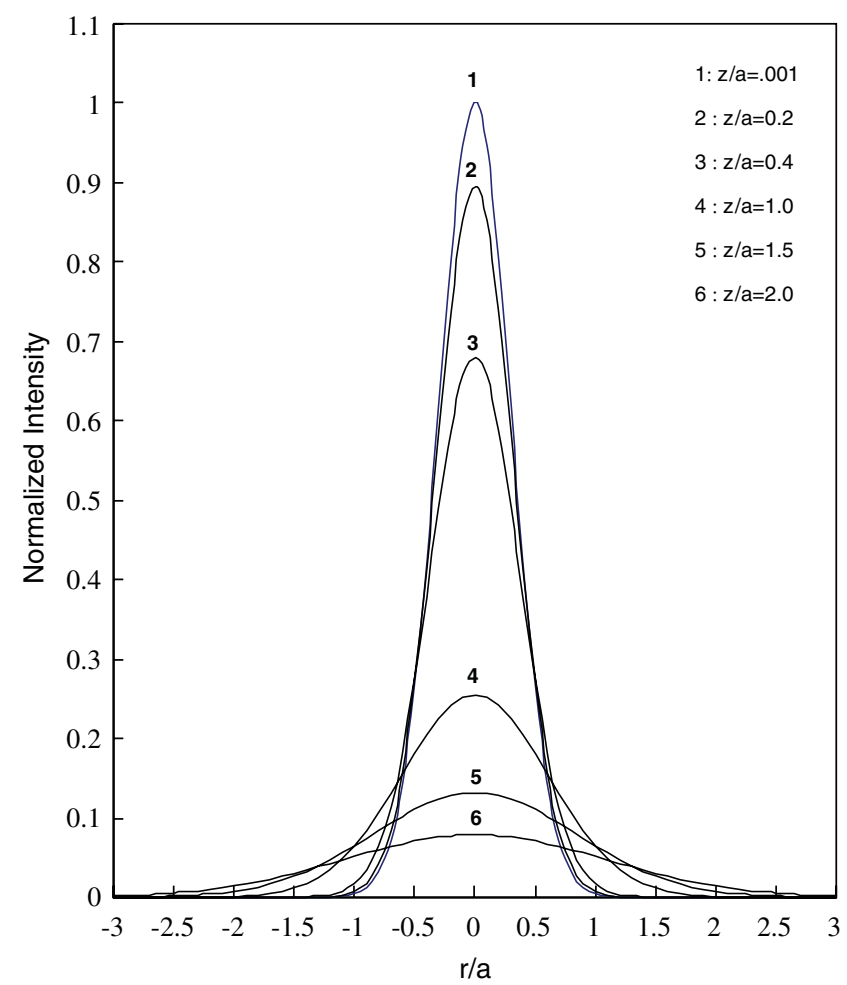

Fig. 3. Simulated light intensity distribution profiles at the nano-aperture tip of fiber probe under illumination mode.
The above-mentioned theoretical model has demonstrated that if we want to obtain the near-field signal, the working distance (WD) between a local probe and a sample surface must be kept in the evanescent field range. The constant distance scanning mode [17] is used in our NSOM system, thus the WD can be controlled at about $5 \mathrm{~nm}$ by the "setpoint" parameter [18]. The following will further state in detail the two theoretical simulation steps of the near-field energy distribution collected by a photodiode underneath the sample.

(1) Fig. 2(a) shows the AFM image of a 2D periodic array of polystyrene spheres. A small region of this sample surface is scanned and its lateral line profile is shown in Fig. 4(a). Defining parameter, $r$, in Eq. (2) as a function of $x$-axis. Integrating the lateral scanned displacement, we get

$$
E_{0}(x)=\int I\left(x^{\prime}-x, z\left(x^{\prime}\right)\right) \mathrm{d} x^{\prime}
$$

(2) Supposing the polystyrene spheres is a kind of chromatic dispersion medium, under the condition of near field, its transmission function [15], $T\left(x^{\prime}\right)$, and absorption function [19], $A\left(x^{\prime}\right)$, are given as

$E_{t}(x)=\int I\left(x^{\prime}-x, z\left(x^{\prime}\right)\right) T\left(x^{\prime}\right) A\left(x^{\prime}\right) \mathrm{d} x^{\prime}$,

where $\mathrm{d} x^{\prime}$ is the thickness of medium that varies with the changes of displacement when lateral scanning across the sample, and $n$ is the refractive index of polystyrene spheres, which is approximately 1.6 , and $\beta$ is known as absorption coefficient. Substituting the above parameters into Eq. (4), the amount of near field energy received by photodiode can then be computed.

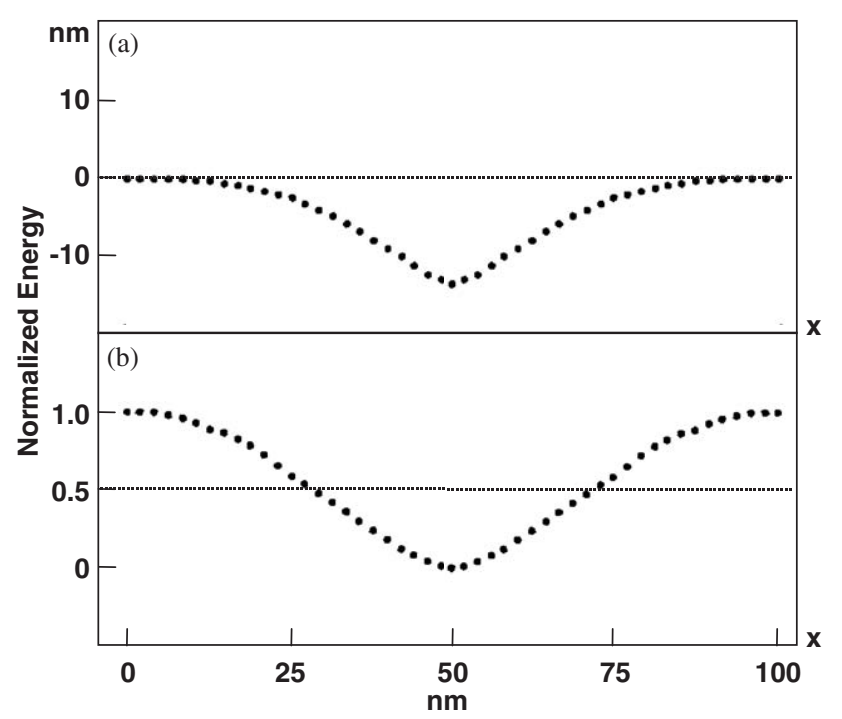

Fig. 4. (a) Line profile as a function of AFM lateral scanning across the $100 \mathrm{~nm}$ polystyrene spheres, (b) normalized energy distribution curve as near field optical signal illuminated at the polystyrene spheres. 


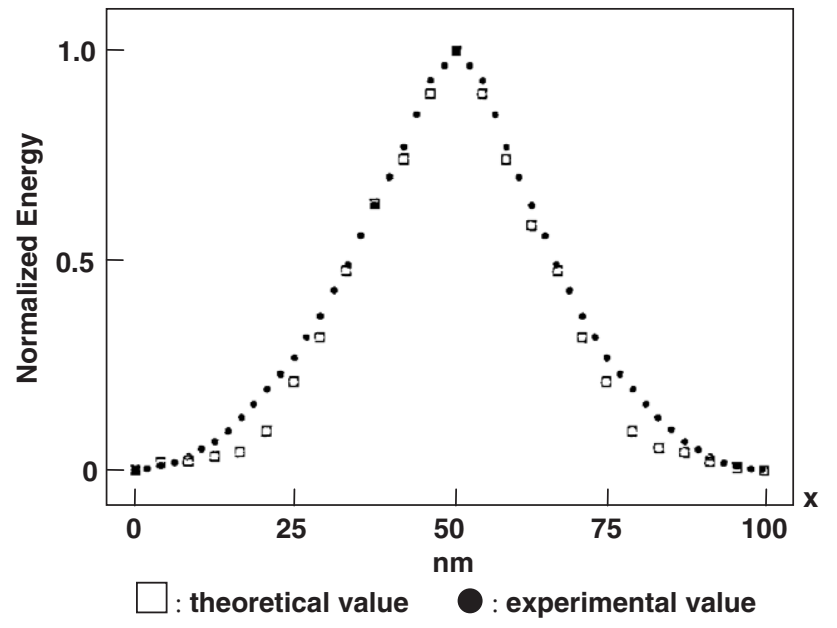

Fig. 5. Simulated energy distribution curve as near field signal penetrates through the $100 \mathrm{~nm}$ polystyrene spheres to photodiode. The absorption coefficient, $\beta$, is fixed at $0.06(1 / \mathrm{nm})$.

Integration is performed over the lateral displacement when scanning across the sample. It is noted that selection of the value of absorption coefficient, $\beta$, contributes a direct impact in errors analysis. After numerous attempts, it is realized that by fixing $\beta$ at $0.06(1 / \mathrm{nm})$, the difference in energy distribution profile between theoretical values and experimental values falls within $5 \%$ range. This is illustrated in Fig. 5, in which, the square and the black dot correspond to theoretical values and experimental values.

\section{Conclusion}

In conclusion, we have shown that with slight modification, a commercial AFM microscope coupled with externally connected signal measurement instruments can be used to acquire high-resolution surface topography and near field optical image concurrently. Furthermore, a complete near field energy distribution theoretical model has been established. During the present nano-technology era, photons, with characteristics of high transmission speed and broad frequency bandwidth, possess a great potential to substitute electrons and become the main transmission carrier in communication sector. The theoretical model we have constructed in this article will be able to determine transmission parameters of near-field photons in different materials. This set up, taking advantage of the high-resolution feature of the near field optics, could be an indispensable analytical technique in nanometer optical communication or even in the bioscience sector.

\section{Acknowledgments}

The authors acknowledge Heh-Nan Lin at National Tsing Hua University and Pan-Ming Ong at Singapore University for technical assistance. This work was supported by the National Science Council of the Republic of China under Grant no. 94-2215-E-415-001.

\section{References}

[1] Sarid Dror. Scanning force microscopy. New York: Oxford University; 1991.

[2] Chen S-H, Chen Y-F. Rev Sci Instrum 2001;72:268.

[3] Chen S-H, Tsai DP, Chen Y-F, Ong P-M. Rev Sci Instrum 1999;70:4463.

[4] Lin H-N, Chen SH, Lee LJ. Rev Sci Instrum 1998;69:3840.

[5] Tsai DP, Li WK. J Vac Sci Technol A 1997;15:1427.

[6] Tsai DP, Guo WR. J Vac Sci Technol A 1997;15:1442.

[7] Betzig E, Finn PL, Weiner JS. Appl Phys Lett 1992;60:2484.

[8] Toledo-Crow R, Yang PC, Chen Y, Vaez-Irarani M. Appl Phys Lett 1992;60:2957.

[9] Shchemelinin A, Rudman M, Lieberman K, Lewis A. Rev Sci Instrum 1993;64:3538.

[10] Garcia-Parajo M, Tate T, Chen Y. Ultramicroscopy 1995;61:155.

[11] Bozhevolnyi SI, Vohnsen B, Bozhevolnaya EA. Opt Commun 1999;172:171.

[12] Rudow O, Vollkopf A, Müller-Wiegand M, Georgiev G, Oesterschulze E. Opt Commun 2001;189:187.

[13] Leviatan Y. J Appl Phys 1986;60:1577.

[14] Wolf E, Nieto-Vesperinas M. J Opt Soc Am 1985;2:886.

[15] Butler DJ, Nugent KA, Roberts A. J Appl Phys 1994;75:2753.

[16] Courjon D, Bainier C. Rep Prog Phys 1994;57:989.

[17] The distance is kept approximately constant by vibrating the tip and measuring the amplitude of vibration.

[18] This parameter defines the amplitude of the voltage applied to the piezo system which drives the cantilever vibration of the fiber probe.

[19] Griffiths DJ. Introduction to electrodynamics. NJ: Prentice-Hall; 1989. 